\title{
ON LELONG-BREMERMANN LEMMA
}

\author{
AYDIN AYTUNA AND VYACHESLAV ZAKHARYUTA
}

(Communicated by Mei-Chi Shaw)

\begin{abstract}
The main theorem of this note is the following refinement of the well-known Lelong-Bremermann Lemma:

Let $u$ be a continuous plurisubharmonic function on a Stein manifold $\Omega$ of dimension $n$. Then there exists an integer $m \leq 2 n+1$, natural numbers $p_{s}$, and analytic mappings $G_{s}=\left(g_{j}^{(s)}\right): \Omega \rightarrow \mathbb{C}^{m}, s=1,2, \ldots$, such that the sequence of functions

$$
u_{s}(z)=\frac{1}{p_{s}} \max \left(\ln \left|g_{j}^{(s)}(z)\right|: j=1, \ldots, m\right)
$$

converges to $u$ uniformly on each compact subset of $\Omega$.

In the case when $\Omega$ is a domain in the complex plane, it is shown that one can take $m=2$ in the theorem above (Section 3); on the other hand, for $n$-circular plurisubharmonic functions in $\mathbb{C}^{n}$ the statement of this theorem is true with $m=n+1$ (Section 4 ). The last section contains some remarks and open questions.
\end{abstract}

\section{INTRODUCTION}

An important consequence of Oka's Theorem about characterization of domains of holomorphy in terms of pseudoconvexity is the result on the coincidence of the class Psh $(D)$ of all plurisubharmonic functions in a pseudoconvex domain with the class of all Hartogs functions in $D$ (Bremermann [5], see also [7, 16]; the onedimensional case has been investigated considerably earlier by Lelong [11]). In equivalent form this result says that every plurisubharmonic function in a pseudoconvex domain $D$ is the regularized upper limit of some sequence $\alpha_{i} \ln \left|f_{i}(z)\right|$ with $f_{i}$ analytic in $D$ and $\alpha_{i}>0$.

An immediate corollary of the above result is the following statement known also as the Lelong-Bremermann Lemma:

Proposition 1. Let $u$ be a continuous plurisubharmonic function on a pseudoconvex domain $D$. Then for each compact subset $K$ of $D$ and $\varepsilon>0$ there exists a natural number $N$, an analytic mapping $F=\left(f_{i}\right): D \rightarrow \mathbb{C}^{N}$, and numbers $\alpha_{i}>0$ such that

$$
\left|u(z)-\max \left\{\alpha_{i} \ln \left|f_{i}(z)\right|: i=1, \ldots ., N\right\}\right|<\varepsilon, \quad z \in K .
$$

For more recent related results of this type in various modes of convergence we refer the reader to [10] and [6]. Proposition 1 does not say anything about the

Received by the editors October 24, 2006 and, in revised form, March 7, 2007.

2000 Mathematics Subject Classification. Primary 32U05; Secondary 31C10.

Key words and phrases. Plurisubharmonic functions, Lelong-Bremermann Lemma. 
behavior of the numbers $N=N(K, \varepsilon)$. However, information about the bound for $N$ is welcomed in certain investigations such as attempts to approximate simultaneously a pluriregular pair by a sequence of similar analytic polyhedral pairs (see $[12,13,18])$.

The main result of this paper (Theorem 1) says that the number $N$ in the above proposition can be taken $\leq 2 n+1$, where $n$ is the dimension of $D$. The proof is based on a generalization of the reduction argument given in [18], Lemma 2, combined with a perturbation argument for smooth mappings.

In Section 3 we examine the one-dimensional case in more detail and prove that for the set of all continuous subharmonic functions the least upper bound of the number of analytic functions involved is just two.

In Section 4 we consider some special classes of plurisubharmonic functions for which the number $N$ can be better estimated. In the last section we give final remarks and discuss some unsolved questions.

\section{MAIN THEOREM}

Theorem 1. Let $u$ be a continuous plurisubharmonic function on a Stein manifold $\Omega$ of dimension $n$. Then there is a sequence of analytic mappings $G_{i}=\left(g_{j}^{(i)}\right)$ : $\Omega \rightarrow \mathbb{C}^{2 n+1}$ and a sequence of natural numbers $p_{i}$ such that the sequence

$$
\frac{1}{p_{i}} \max \left\{\ln \left|g_{j}^{(i)}(z)\right|: j=\overline{1,2 n+1}\right\}, i \in \mathbb{N}
$$

converges to $u(z)$ uniformly on each compact subset of $\Omega$.

Proof. Fix a compact subset $K$ of $\Omega$ and $\delta>0$. In view of the Lelong-Bremermann Lemma we can find analytic on $\Omega$ functions $f_{j}$ and $\alpha_{j}>0, j=1, \ldots, N$ such that

$$
u(z)-\frac{\delta}{4} \leq v(z) \leq u(z), \quad z \in K
$$

where $v(z):=\max \left\{\alpha_{j} \ln \left|f_{j}(z)\right|: j=\overline{1, N}\right\}$. The natural number $N$, in general, depends upon $K$ and $\delta$. Since $v(z)$ is continuous on $K$, we can assume, without loss of generality, that $\alpha_{j}=\frac{1}{q}, 1 \leq j \leq N$ with some natural number $q$.

Set $k=2 n+2$ and suppose that $N \geq k$. Consider the set $\mathcal{J}_{k}$ of all $k$-tuples $J=\left(j_{1}, \ldots, j_{k}\right)$ such that $1 \leq j_{1}<\ldots<j_{k} \leq N$ and introduce the set

$$
\Delta^{k}:=\left\{w=\left(w_{\nu}\right) \in \mathbb{C}^{k}:\left|w_{1}\right|=\ldots=\left|w_{\nu}\right|=\ldots=\left|w_{k}\right|\right\} .
$$

For each $J=\left(j_{\nu}\right) \in \mathcal{J}_{k}$ we define the mapping $\Phi_{J}: \Omega \times \Delta^{k} \rightarrow \mathbb{C}^{k}$ by the formula $\Phi_{J}(z, w):=\left(f_{j_{\nu}}(z)-w_{\nu}\right)$. Since the real dimension of the manifold $\Omega \times \Delta^{k}$ is $2 n+k+1$, in view of Sard's Theorem, the closed set $\Phi_{J}\left(K \times \Delta^{k}\right)$ in $\mathbb{C}^{k}=\mathbb{R}^{2 k}$ has Lebesgue measure zero and hence is nowhere dense in $\mathbb{C}^{k}$. Therefore all the sets

$$
S_{J}:=\left\{\zeta=\left(\zeta_{j}\right) \in \mathbb{C}^{N}:\left(\zeta_{j_{v}}\right) \in \Phi_{J}\left(K \times \Delta^{k}\right)\right\}, J=\left(j_{\nu}\right) \in \mathcal{J}_{k},
$$

are closed and nowhere dense in $\mathbb{C}^{N}$. So the set $S=\bigcup_{J \in \mathcal{J}_{k}} S_{J}$ is also closed and nowhere dense in $\mathbb{C}^{N}$. Thus for each $\varepsilon>0$ there is $\eta=\left(\eta_{j}\right) \in \mathbb{C}^{N} \backslash S$ with

$$
\max \left\{\left|\eta_{j}\right|: j=\overline{1, N}\right\}<\varepsilon .
$$

Then the mapping $h=\left(h_{j}\right):=\left(f_{j}+\eta_{j}\right)$ has the property:

$$
\left\{z \in K:\left|h_{j_{1}}(z)\right|=\ldots=\left|h_{j_{\nu}}(z)\right|=\ldots=\left|h_{j_{k}}(z)\right|\right\}=\varnothing
$$


for every $J=\left(j_{\nu}\right) \in \mathcal{J}_{k}$. Due to (2.3), (2.2), one can choose $\varepsilon$ sufficiently small to provide the estimate

$$
|u(z)-w(z)|<\frac{\delta}{2}, z \in K,
$$

where $w(z):=\frac{1}{q} \max \left\{\ln \left|h_{j}(z)\right|: 1 \leq j \leq N\right\}$.

Now set $m=k-1=2 n+1$. The property (2.4) helps us to use an idea from [18] on how to reduce the number of functions from $N$ to $m$. Namely, we construct a sequence of mappings $h^{(s)}=\left(h_{r}^{(s)}\right)_{r=1}^{m} \in A(\Omega)^{m}, s \in \mathbb{N}$, by the formula

$$
h_{r}^{(s)}(z):=\sum_{J=\left(j_{\nu}\right) \in \mathcal{J}_{r}}\left(h_{j_{1}}(z) \cdot \ldots \cdot h_{j_{r}}(z)\right)^{s \frac{m !}{r}}, 1 \leq r \leq m
$$

and consider the sequence of functions

$$
w_{s}(z):=\frac{1}{q s m !} \max \left(\ln \left|h_{r}^{(s)}(z)\right|: r=1, \ldots, m\right), s \in \mathbb{N} .
$$

We shall show that there is an $S_{0}>0$ such that

$$
\left|u(z)-w_{s}(z)\right|<\delta, z \in K, s \geq S_{0} .
$$

It is easily seen that $\left|h_{r}^{(s)}(z)\right| \leq 2^{N} \max \left\{\left|h_{j}(z)\right|^{s m !}: 1 \leq j \leq N\right\}$. Hence, taking into account (2.5), we get the estimate from above

$$
w_{s}(z) \leq u(z)+\frac{\delta}{2}+\frac{N \ln 2}{q s m !} \leq u(z)+\delta, \quad z \in K, s \geq S_{1}
$$

with some $S_{1}>0$. Now we will estimate the sequence (2.7) from below. Fix $z \in K$. Then there is $r=r(z) \leq m$ and $J=\left(j_{\nu}\right) \in \mathcal{J}_{r}$ such that

$$
\left|h_{j_{1}}(z)\right|=\ldots=\left|h_{j_{r}}(z)\right|>\left|h_{i}(z)\right|, i \notin J .
$$

We choose an open neighborhood $U_{z} \Subset \Omega$ of $z$ so that

$$
d(z):=\max _{I} \sup _{\zeta \in U_{z}}\left\{\left|\frac{h_{i_{1}}(\zeta) \cdot \ldots \cdot h_{i_{r}}(\zeta)}{h_{j_{1}}(\zeta) \cdot \ldots \cdot h_{j_{r}}(\zeta)}\right|\right\}<1,
$$

where the outer maximum is taken over all $r$-tuples $I=\left(i_{\nu}\right) \in \mathcal{J}_{r}, I \neq J$. By continuity, we can suppose also that $U_{z}$ is such that the conditions

$$
|w(z)-w(\zeta)|<\sigma, \quad(1-\sigma)\left|h_{j}(z)\right|<\left|h_{j}(\zeta)\right|, \quad \zeta \in U_{z}, \quad j \in J
$$

hold with $\sigma>0$ (this number will be chosen later). Then the inequality

$$
\begin{aligned}
\left|h_{r}^{(s)}(\zeta)\right| & \geq\left|h_{j_{1}}(\zeta) \cdot \ldots \cdot h_{j_{r}}(\zeta)\right|^{\frac{s m !}{r}}\left(1-\sum_{I \neq J}\left|\frac{h_{i_{1}}(\zeta) \cdot \ldots \cdot h_{i_{r}}(\zeta)}{h_{j_{1}}(\zeta) \cdot \ldots \cdot h_{j_{r}}(\zeta)}\right|^{s}\right)^{\frac{m !}{r}} \\
& \geq\left((1-\sigma)\left|h_{j_{1}}(z)\right|\right)^{s m !}\left(1-2^{N} d(z)^{s}\right)^{\frac{m !}{r}}
\end{aligned}
$$

holds for all $\zeta \in U_{z}$ with $r=r(z)$. Thus, for $\zeta \in U_{z}$, we have

$$
\begin{aligned}
w_{s}(\zeta) & =\frac{1}{q s m !} \max \left(\ln \left|h_{j}^{(s)}(\zeta)\right|: j=\overline{1, m}\right) \geq \frac{1}{q s m !} \ln \left|h_{r}^{(s)}(\zeta)\right| \\
& \geq \frac{1}{q}\left(\ln (1-\sigma)+\ln \left|h_{j_{1}}(z)\right|\right)+\frac{1}{q s r} \ln \left(1-2^{N} d(z)^{s}\right)
\end{aligned}
$$


for sufficiently large $s$. Since $w(z)=\frac{1}{q} \ln \left|h_{j_{1}}(z)\right| \geq w(\zeta)-\sigma$, we can choose $\sigma=\sigma(z)$ and $S=S(z)$ so that

$$
w_{s}(\zeta) \geq w(\zeta)-\delta / 2, \quad \zeta \in U_{z}, \quad s \geq S .
$$

A compactness argument together with (2.5) now gives $S_{2}$ such that

$$
w_{s}(\zeta) \geq w(\zeta)-\delta / 2 \geq u(\zeta)-\delta, \zeta \in K, s \geq S_{2} .
$$

Taking into account (2.9), this yields (2.8) with $S_{0}=\max \left\{S_{1}, S_{2}\right\}$. Hence, setting $g_{j}:=h_{j}^{(s)}, j=1, \ldots, m$, and $p=q s m$ ! with some $s \geq S_{0}$, we obtain an analytic mapping $G=\left(g_{j}\right)_{j=1}^{2 n+1}: \Omega \rightarrow \mathbb{C}^{2 n+1}$ and a natural number $p$ such that

$$
\sup _{z \in K}\left|u(z)-\frac{1}{p} \max \left(\ln \left|g_{j}(\zeta)\right|: j=\overline{1,2 n+1}\right)\right| \leq \delta .
$$

Now consider an exhaustion of the Stein manifold by compact sets $\left\{K_{i}\right\}_{i=1}^{\infty}$ and a sequence of positive numbers $\left\{\delta_{i}\right\}_{i=1}^{\infty}$ that converges to zero. Let $G_{i}=\left(g_{j}^{(i)}\right)$ : $\Omega \rightarrow \mathbb{C}^{2 n+1}$ and $p_{i}$ be constructed as above for $K=K_{i}$ and $\delta=\delta_{i}, i \in \mathbb{N}$. Then, due to (2.10), the sequence (2.1) converges to $u$ uniformly on each compact subset of the Stein manifold $\Omega$.

\section{Approximation of subharmonic functions}

Here we show that in the one-dimensional case, Theorem 1 is true with $N=2$. First we consider two lemmas. During the preparation of this paper for publication, we became aware of a result (see, preprint [3], Theorem 1.2) that is somehow stronger than Lemma 1 below. We decided to keep our proof of this lemma since it is more direct and does not use the Yulmukhamedov Lemma (see, e.g., [3], Lemma A).

Lemma 1. Suppose that $\mu$ is a positive Borel measure with a compact support $K$ and that the potential

$$
v(z):=\int_{K} \ln |\zeta-z| d \mu(\zeta)
$$

is continuous on $\mathbb{C}$. Then there exist sequences of polynomials $P_{s}$ and $Q_{s}$ of a common degree $N_{s}$ such that the sequence

$$
v_{s}(z):=\frac{M}{N_{s}} \max \left\{\ln \left|P_{s}(z)\right|, \ln \left|Q_{s}(z)\right|\right\},
$$

where $M=\mu(K)$, converges to $v(z)$ uniformly on $\mathbb{C}$.

Proof. Without loss of generality we suppose that $v \in C^{2}(D)$; hence $\mu=w d \lambda$ where $w$ is a continuous function in $\mathbb{C}$, vanishing outside of $K$, and $\lambda$ is the Lebesgue measure on $\mathbb{C}=\mathbb{R}^{2}$. Let $d_{s}:=2^{-s}$. Given $s \in \mathbb{N}$ and $\alpha=\left(\alpha_{1}, \alpha_{2}\right) \in \mathbb{Z}^{2}$ we denote by $\Delta_{s}(\alpha)$ the square

$$
\left\{x+i y: \alpha_{1} d_{s}<x \leq\left(\alpha_{1}+1\right) d_{s} ; \alpha_{2} d_{s}<y \leq\left(\alpha_{2}+1\right) d_{s}\right\}
$$

and set $\Lambda_{s}(\alpha)=\frac{1+i}{2^{s+1}}+\Delta_{s}(\alpha)$. Let $a_{s}(\alpha)$ be a center of the square $(3.3), b_{s}(\alpha)$ its upper-right vertex (that is, the center of the square $\Lambda_{s}(\alpha)$ ) and $A_{s}$ be the set of all $\alpha \in \mathbb{Z}^{2}$ provided that the distance of the square $\Delta_{s}(\alpha)$ from $K$ does not exceed $2 d_{s}$. The last assumption implies the conditions:

$$
K \cap \Delta_{s}(\alpha)=K \cap \Lambda_{s}(\alpha)=\varnothing \text {, if } \alpha \notin A_{s} .
$$


For $\alpha \in A_{s}$ we choose nonnegative integers $m_{s}(\alpha)$ and $n_{s}(\alpha)$ so that the inequalities

$$
\left|M m_{s}(\alpha)-8^{s} \mu\left(\Delta_{s}(\alpha)\right)\right| \leq M ;\left|M n_{s}(\alpha)-8^{s} \mu\left(\Lambda_{s}(\alpha)\right)\right| \leq M
$$

hold with $M=\mu(K)$ and $\sum m_{s}(\alpha)=\sum n_{s}(\alpha)=8^{s}$. We show that the sequences of polynomials

$$
P_{s}(z)=\prod_{\alpha \in A_{s}}\left(z-a_{s}(\alpha)\right)^{m_{s}(\alpha)} ; Q_{s}(z)=\prod_{\alpha \in A_{s}}\left(z-b_{s}(\alpha)\right)^{n_{s}(\alpha)}
$$

are sought-for ones with $N_{s}=8^{s}$.

We introduce $E_{s}$ (respectively, $F_{s}$ ) as a set of all points $z \in \mathbb{C}$ with the distance $\geq$ $2^{-(s+2)}$ from all zeros of the polynomial $P_{s}$ (respectively, $Q_{s}$ ). By the construction we have $E_{s} \cup F_{s}=\mathbb{C}$.

Using the notation $p_{s}(z):=\frac{M}{N_{s}} \ln \left|P_{s}(z)\right|$, we want to show that

$$
\left|v(z)-p_{s}(z)\right| \leq \varepsilon(s), z \in E_{s}
$$

with $\varepsilon(s) \rightarrow 0$ as $s \rightarrow \infty$.

First we prove this estimate with $\widetilde{p}_{s}(z):=\sum_{\alpha \in A_{s}} \mu\left(\Delta_{s}(\alpha)\right) \ln \left|a_{s}(\alpha)-z\right|$ instead of $p_{s}(z)$. Fix $z \in E_{s}$ and introduce the notation:

$$
A_{s}^{\prime}:=\left\{\alpha \in A_{s}:\left|a_{s}(\alpha)-z\right| \geq \sqrt{d_{s}}\right\}, A_{s}^{\prime \prime}:=A_{s} \backslash A_{s}^{\prime}, B_{s}:=\bigcup_{\alpha \in A_{s}^{\prime \prime}} \Delta_{s}(\alpha) .
$$

Then

$$
\begin{aligned}
\left|v(z)-\widetilde{p}_{s}(z)\right| & \leq \sum_{\alpha \in A_{s}} \int_{\Delta_{s}(\alpha)}|\ln | \zeta-z|-\ln | a_{s}(\alpha)-z|| d \mu(\zeta) \\
& \leq I_{1}+I_{2}+I_{3}
\end{aligned}
$$

where

$$
\begin{gathered}
I_{1}:=-\sum_{\alpha \in A_{s}^{\prime}} \int_{\Delta_{s}(\alpha)} \ln \left(1-\frac{\left|\zeta-a_{s}(\alpha)\right|}{\left|a_{s}(\alpha)-z\right|}\right) d \mu(\zeta), \\
I_{2}:=C \int_{B_{s}}|\ln | \zeta-z|| d \lambda(\zeta), \\
I_{3}:=C \lambda\left(B_{s}\right) \max \left\{|\ln | a_{s}(\alpha)-z||: z \in B_{s}\right\}
\end{gathered}
$$

with $C:=\max \{w(z): z \in K\}$, where $w$ is defined in the very beginning of the proof. Since $\left|\zeta-a_{s}(\alpha)\right| \leq d_{s}<1 / 2$ if $\zeta \in \Delta_{s}(\alpha)$, we have $I_{1} \leq 2 M \sqrt{d_{s}}=$ : $\varepsilon_{1}(s)$. On the other hand, $I_{2} \leq 2 \pi C \int_{0}^{2 \sqrt{d_{s}}} \rho|\ln \rho| d \rho=: \varepsilon_{2}(s)$. Finally, due to the definition of $E_{s}$, we have $\left|a_{s}(\alpha)-z\right| \geq d_{s} / 4$; therefore $I_{3} \leq 4 \pi C d_{s}(s+2) \ln 2=$ : $\varepsilon_{3}(s)$.

Now we set $R_{s}:=4^{s} \max \{|z|: z \in K\}$. Taking into account that $\# A_{s} \leq C^{\prime} 4^{s}$ with some constant $C^{\prime}$ and applying (3.5), we obtain the estimate:

$$
\begin{aligned}
\left|p_{s}(z)-\widetilde{p}_{s}(z)\right| & \leq \ln R_{s} \sum_{\alpha \in A_{s}}\left|\mu\left(\Delta_{s}(\alpha)\right)-\frac{M m_{s}(\alpha)}{8^{s}}\right| \\
& \leq M C C^{\prime} 4^{-s} \ln R_{s}=: \varepsilon_{4}(s)
\end{aligned}
$$

for all $z \in E_{s}$ such that $|z| \leq R_{s}$. Combining the above estimates, we obtain that the estimate (3.6) holds for all $z \in E_{s}$ such that $|z| \leq R_{s}$ with $\varepsilon(s):=\sum_{j=1}^{4} \varepsilon_{j}(s)$, which tends to 0 as $s \rightarrow \infty$. 
The function $\psi(z):=v(z)-p_{s}(z)$ is harmonic for $|z|>R_{s}$ and $\psi(z)=c \ln z+$ $h(z)$, where $h(z)$ is harmonic at $\infty$. But

$$
c=\mu(K)\left(1-8^{-s} \sum_{\alpha \in L_{s}} m_{s}(\alpha)\right)=0 .
$$

Therefore, by the maximum principle, the estimate (3.6) is true also if $|z|>R_{s}$. In the same way one can prove the estimate

$$
\left|v(z)-q_{s}(z)\right| \leq \delta(s), z \in F_{s}
$$

for $q_{s}(z):=\frac{M}{N_{s}} \ln \left|Q_{s}(z)\right|$ with $\delta(s) \rightarrow 0$ (we may assume that $\varepsilon(s)=\delta(s)$ ). Then, combining (3.6), (3.7), we obtain that

$$
q_{s}(z)-2 \varepsilon(s) \leq p_{s}(z) \leq q_{s}(z)+2 \varepsilon(s), z \in E_{s} \cap F_{s} .
$$

Moreover, due to the maximum principle, the right inequality in (3.8) is true also on every $\operatorname{disc}\left\{\left|z-a_{s}(\alpha)\right|<d_{s} / 4\right\}$, while the left one is so in every disc $\left\{\left|z-b_{s}(\alpha)\right|<d_{s} / 4\right\}$. Since $v_{s}(z)=\max \left\{p_{s}(z), q_{s}(z)\right\}$, we obtain that the estimate $\left|v(z)-v_{s}(z)\right| \leq 3 \varepsilon(s)$ holds for all $z \in \mathbb{C}$, which completes the proof.

Lemma 2. Let $\Omega$ be a domain in $\widehat{\mathbb{C}}$ with the boundary $\partial \Omega$ formed by a finite number of disjoint smooth Jordan curves and $M=\left\{a_{1}, \ldots, a_{k}, \ldots, a_{m}\right\}$ be a finite subset of $\widehat{\mathbb{C}} \backslash \Omega$, containing just one point from each connected component. Suppose that $\bar{\varphi}$ is a continuous function on $\Omega$, which is harmonic in $\Omega$. Then for each $\delta>0$ there is a function $g \in A(\widehat{\mathbb{C}} \backslash M)$, such that $g(z) \neq 0$ in $\widehat{\mathbb{C}} \backslash \Omega$ and a natural number $q$ such that

$$
\left|\varphi(z)-\frac{1}{q} \ln \right| g(z)||<\delta, z \in \bar{\Omega} .
$$

Proof. By Keldysh's approximation theorem (see, e.g., [2], 7.9), for each $\delta>0$ there is a function $\psi(z)$ harmonic in $\widehat{\mathbb{C}} \backslash M$ and such that

$$
|\varphi(z)-\psi(z)|<\delta / 2, z \in \bar{\Omega} .
$$

On the other hand, due to the Logarithmic Conjugation Theorem (see, e.g., [1], 9.15), there exist $s \in A(\widehat{\mathbb{C}} \backslash M)$ and real numbers $b_{1}, \ldots, b_{k}, \ldots, b_{m}$ such that

$$
\psi(z)=\operatorname{Res}(z)+b_{1} \ln \left|z-a_{1}\right|+\ldots+b_{m} \ln \left|z-a_{m}\right|
$$

for all $z \in \widehat{\mathbb{C}} \backslash M$. Now, choosing integers $p_{k}$ and $q \in \mathbb{N}$ so that

$$
\max \left\{\sum_{k=1}^{m}\left|b_{k}-\frac{p_{k}}{q}\right||\ln | z-a_{m}||: z \in \bar{\Omega}\right\}<\frac{\delta}{2},
$$

we obtain (3.9) with $g(z)=\left(z-a_{1}\right)^{p_{1}} \cdot \ldots \cdot\left(z-a_{1}\right)^{p_{m}} \exp p s(z)$.

Theorem 2. Let $u$ be a continuous subharmonic function in a domain $D \subset \widehat{\mathbb{C}}$, $D \neq \widehat{\mathbb{C}}$. Then for any compact subset $K \subset D$ and each $\varepsilon>0$ there exist functions $f_{1}, f_{2}$, analytic in $D$, and $\alpha>0$ such that

$$
\left|u(z)-\alpha \max \left\{\ln \left|f_{1}(z)\right|, \ln \left|f_{2}(z)\right|\right\}\right|<\varepsilon, z \in K .
$$


Proof. We may assume that $\infty \notin D$; otherwise we apply a change of variables $w=\frac{1}{z-a}$ with $a \notin D$. On the other hand, we may suppose, without loss of generality, that $K=\bar{\Omega}$, where $\Omega$ is a domain with the boundary $\partial \Omega$ formed by a finite number of disjoint smooth Jordan curves. By the Riesz Representation Theorem (see, e.g., [14]) there exist a unique Borel measure $\mu$ in $D$ and a function $\varphi \in h(\Omega)$ such that

$$
u(z)=\int_{K} \ln |\zeta-z| d \mu(\zeta)+\varphi(z), z \in \Omega .
$$

It is easy to see that the function $v(z):=\int_{K} \ln |\zeta-z| d \mu(\zeta)$ is continuous on $K$, hence (by [9], Theorem 5.1) in $C$. So, the function $\varphi$ is continuously extendable onto $K$. Therefore, applying Lemmas 1,2 with $\delta=\varepsilon / 2$, we choose $\alpha>0$, a couple of polynomials $(P, Q)$ and a function $g \in A(D)$, so that the relation (3.10) will be fulfilled if we set

$$
f_{1}(z):=P(z) e^{\alpha g(z)} ; \quad f_{2}(z):=Q(z) e^{\alpha g(z)},
$$

which completes the proof.

\section{Approximation of $n$-CIRCUlar Plurisubharmonic FUnCtions}

Let $D$ be a pseudoconvex $n$-circular (Reinhardt) domain in $\mathbb{C}^{n}$. Any plurisubharmonic function $u: D \rightarrow[-\infty, \infty)$ depending only on moduli of coordinates (we will call such functions $n$-circular) is convex with respect to the variables $t_{\nu}=\ln \left|z_{\nu}\right|$. It is easily seen that this function can be represented in the form

$$
u(z)=\sup \left\{\sum_{\nu=1}^{n} \alpha_{\nu} \ln \left|z_{\nu}\right|-\beta:\left(\alpha_{1}, \ldots, \alpha_{\nu}, \ldots, \alpha_{n} ; \beta\right) \in M\right\}
$$

with some set $M=M(u) \subset \mathbb{R}^{n+1}$. Notice that if $D$ is complete, then the $\alpha_{\nu}$ are always nonnegative in (4.1).

Theorem 3. Let u be a continuous n-circular plurisubharmonic function in a pseudoconvex n-circular domain $D$. Then there exists a sequence of rational mappings $Q^{(r)}=\left(Q_{j}^{(r)}\right): \mathbb{C}^{n} \rightarrow \mathbb{C}^{n+1}$ such that the sequence

$$
u_{r}(z)=\max \left\{\ln \left|Q_{j}^{(r)}(z)\right|: j=\overline{1, n+1}\right\}, \quad r \in \mathbb{N}
$$

converges to $u(z)$ uniformly on each compact subset of $D$. If the domain $D$ is complete, then all $Q_{j}^{(r)}$ are polynomial.

Proof. Fix any compact set $K \subset D$. Since $u$ is continuous, we conclude from the representation (4.1), following [18], that for each $\delta>0$ there is a function

$$
v(z)=\frac{1}{q} \sup \left\{\sum_{\nu=1}^{n} m_{\nu, j} \ln \left|z_{\nu}\right|+\beta_{j}: j=\overline{1, N}\right\},
$$

where $m_{\nu, j}$ are integers and $q$ is a natural number, such that

$$
|u(z)-v(z)|<\delta, z \in K .
$$

We will use the notation

$$
l_{j}(t):=\sum_{\nu=1}^{n} m_{\nu, j} \ln t_{\nu}+\beta_{j}, \quad \mathbb{R}_{+}^{n}
$$


where $t=\left(t_{\nu}\right) \in \mathbb{R}_{+}^{n}=[0,+\infty)^{n}$.

Now we want to choose numbers $\xi_{j}$ so that $\left|\xi_{j}\right|<\delta, j=\overline{1, N}$, and for each $z \in D$ the supremum in the expression

$$
w(z):=\frac{1}{q} \sup \left\{l_{j}\left(\left(\left|z_{\nu}\right|\right)\right)-\xi_{j}: j=\overline{1, N}\right\}
$$

is attained for no more than $n+1$ indices $j$. Set $k=n+2$ and denote by $\mathcal{J}_{k}$ the set of all $k$-tuples $J=\left\{j_{1}, \ldots, j_{k}\right\}$ such that $1 \leq j_{1}<\ldots<j_{k} \leq N$. Given $J \in \mathcal{J}_{k}$, we set $\Omega_{J}:=\left\{t \in \mathbb{R}_{+}^{n}: l_{j}(t)>-\infty, j \in J\right\}$ and introduce the real analytic mapping $\Phi_{J}: \Omega_{J} \times \mathbb{R} \rightarrow \mathbb{R}^{k}$ so that $\Phi_{J}(t, \tau):=\left(l_{j_{s}}(t)-\tau\right)_{s=1}^{k}$. Then, applying the considerations similar to those used in the proof of Theorem 1, we conclude that there is a nowhere dense set $S \subset \mathbb{R}^{N}$ such that for each $J \in \mathcal{J}_{k}$ and every $t \in \Omega_{J}$ there are at least two different among the numbers $l_{j}(t)-\xi_{j}, j \in J$ for any choice of $\left(\xi_{j}\right) \in \mathbb{R}^{N} \backslash S$; in particular, we can assume that $\left|\xi_{j}\right|<\delta$ for all $j$. Taking into account that the supremum in $(4.3)$ can be attained only if $l_{j}\left(\left(\left|z_{\nu}\right|\right)\right)>-\infty$, we conclude now that for any $z \in D$ the supremum in (4.5) is attained for no more than $n+1$ indices $j$.

The function (4.5) can be written in the form

$$
w(z)=\frac{1}{q} \sup \left\{\ln \left|h_{j}(z)\right|: j=\overline{1, N}\right\},
$$

where $h_{j}(z):=e^{\beta_{j}-\xi_{j}} \prod_{j=1}^{n} z_{\nu}^{m_{\nu, j}}, j=\overline{1, N}$, are analytic in $D$. Now, applying the construction (2.6) with $m=n+1$ to these functions we obtain the sequence (2.7) converging to the function $w(z)$ uniformly on the compact $K$. Finally, taking a sequence of compact sets $K_{r}$ exhausting $D, \delta_{r} \rightarrow 0$ and choosing properly $s=$ $s(r)$, we obtain the desired sequence $u_{r}(z)$ with rational functions $Q_{j}^{(r)}(z)$, by the construction. If the domain $D$ is complete, then in the above considerations all integers $m_{\nu, j}$ must be nonnegative; therefore the functions $h_{j}(z)$ and hence the functions $Q_{j}^{(r)}(z)$ will be polynomials.

Using the representation of Green pluripotential as a plurisubharmonic $n$-circular function ([17], Proposition 1.4.3) yields

Corollary 1. Suppose that $K$ is a pluriregular polynomially convex n-circular compact subset of a logarithmically convex bounded n-circular domain $D$. Then the Green pluripotential

$$
\omega(z):=\limsup _{\zeta \rightarrow z}\left\{\sup \left\{u(\zeta): u \in P \operatorname{sh}(D) ;\left.u\right|_{K} \leq 1 ; u<1 \text { in } D\right\}\right\}
$$

can be approximated by some sequence (4.2), with polynomial mappings $Q^{(r)}$, uniformly on any compact subset of $D$.

\section{Final Remarks}

5.1. The following problem arises in connection with Theorems 1, 2 .

Problem 1. What is the smallest upper bound for $N$ that works for the class of all continuous plurisubharmonic functions on a given manifold?

Naturally, such a bound is expected to be $\leq 2 n$, but in order to prove that, some more delicate methods are needed. For certain specific classes of plurisubharmonic functions the technique used here might yield more refined bounds as was shown in the previous section. 
5.2. Another important example has been considered in [18]: using a construction similar to (2.6) in a combination with some algebraic considerations (see Lemma 6 there), it is shown there that, in the conditions of Corollary 1, the function (4.6) can be approximated uniformly on any compact subset of $D \backslash K$ by a sequence

$$
u_{r}(z)=\frac{1}{q_{r}} \ln \max \left\{\left|Q_{j}^{(r)}(z)\right|: j=\overline{1, n}\right\}, \quad r \in \mathbb{N},
$$

with polynomial mappings $\left(Q_{j}^{(r)}\right): \mathbb{C}^{n} \rightarrow \mathbb{C}^{n}$ and natural numbers $q_{r}$. A further reduction of the number of polynomials $Q_{j}^{(r)}$ to $n=\operatorname{dim} D$ is due to the fact that the pluripotential (4.6) is a maximal plurisubharmonic function ([15]) in the annulus $D \backslash K$.

5.3. Suppose that a sequence

$$
u_{r}(z)=\frac{1}{q_{r}} \ln \max \left\{\left|f_{j}^{(r)}(z)\right|: j=\overline{1, n}\right\}, \quad r \in \mathbb{N},
$$

with $f_{j}^{(r)}$ analytic on a given Stein manifold $D$ and $q_{r} \in \mathbb{N}$, converges uniformly on each compact subset of a subdomain $G \subset D$ to a continuous function $u(z)$. Then it is easily seen that $u$ must be a maximal plurisubharmonic function in $D$, that is, $\left(d d^{c} u(z)\right)^{n} \equiv 0$ in $D([4,15])$. In connection with section 5.2 the following general question arises.

Problem 2. Does the above approximation property characterize the maximality of a continuous plurisubharmonic function? In other words, given a Stein manifold $D$ and a continuous function $u \in P \operatorname{sh}(D)$ such that $\left(d d^{c} u(z)\right)^{n} \equiv 0$ in a domain $G \subset D$, does there exist a sequence (5.1) with $f_{j}^{(r)} \in A(D)$, converging uniformly on each compact subset of $G$ to the function $u(z)$ ?

As follows from Lemma 2, the question is answered positively for any plane domain. For several variables the positive answer to this question is known only in some particular cases, such as that considered in section 5.2.

5.4. Let $u$ be a plurisubharmonic function on a Stein manifold $\Omega, \operatorname{dim} \Omega=n$, which can be represented in the form $u(z)=\lim \sup _{\xi \rightarrow z} \limsup _{s \rightarrow \infty} \frac{\ln \left|h_{s}(\xi)\right|}{q_{s}}, \quad z \in \Omega$ with $q_{s}>0$ and $h_{s}$ from a given subalgebra $L$ of $A(\Omega)$ that contains the constants. Then Proposition 1 is true with all the functions $f_{i}$ belonging to the algebra $L$. Examining the proof of Theorem 1, one can see that all the functions $g_{j}^{(s)}$ in $(2.1)$ will belong to the algebra $L$ as well. Therefore Theorem 1.2 of Gamelin-Sibony [8], combined with such a version of Theorem 1, yields

Theorem 4. Let $D$ be a bounded domain in $\mathbb{C}^{n}$ with smooth boundary, such that $\bar{D}$ has a Stein neighborhood basis. Let $u$ be a real-valued continuous function on $\bar{D}$ that is plurisubharmonic on $D$. Then $u$ can be approximated uniformly on $\bar{D}$ by functions of the form $\max \left\{\alpha_{\nu} \ln \left|g_{\nu}\right|: \nu=\overline{1,2 n+1}\right\}$, where $\alpha_{\nu}>0$ and the $g_{\nu}$ are analytic in some neighborhood of $\bar{D}, \nu=\overline{1,2 n+1}$.

\section{ACKNOWLEDGMENTS}

We are extremely thankful to our referee for very useful remarks. 


\section{REFERENCES}

[1] Axler, S., Bourdon, P., and Ramey, W., Harmonic Function Theory, Springer, 2001 MR1805196 (2001j:31001)

[2] Armitage, D. H. and Gardiner, S. J., Classical Potential Theory, Springer, 2001 MR1801253 (2001m:31001)

[3] Bloom, T., Levenberg, N., and Lyubarskii, Yu., A Hilbert Lemniscate Theorem in $\mathbb{C}^{2}$, preprint

[4] Bedford, E. and Taylor, B. A., The Dirichlet problem for a complex Monge-Ampère equation, Invent. Math., 1976, 37, 1-44 MR0445006 (56:3351)

[5] Bremermann, M. J., On the conjecture of the equivalence of the plurisubharmonic functions and the Hartogs functions, Math. Ann., 1956, 131, 76-86 MR0077644 (17:1070h)

[6] Demailly, J.-P., "On the Ohsawa-Takegoshi-Manivel $L^{2}$ extension theorem", Complex Analysis and Geometry, Progr. Math., 188, Birkhäuser Verlag, Basel, 2000, 47-82 MR1782659 (2001m:32041)

[7] Gunning, R., Introduction to Holomorphic Functions of Several Variables, Vol. 1, Wadsworth and Brooks-Cole, Pacific Grove, CA, 1990 MR1052649 (92b:32001a)

[8] Gamelin, T. W. and Sibony, N., Subharmonicity for uniform algebras, Journal Funct. Anal., 1980, 35, 64-108 MR560218 (81f:46062)

[9] Hayman, W. K. and Kennedy, P. B., Subharmonic Functions, Academic Press, London-New York-San Francisco, 1976 MR0460672 (57:665)

[10] Hörmander, L., Notions of Convexity, Progress in Mathematics, 127, Birkhäuser Verlag, Basel, 1994 MR1301332 (95k:00002)

[11] Lelong, P., Sur quelques problèmes de la théorie des fonctions de deux variables complexes, Ann. Sci. Ecole Norm. Sup., 1941, 58, 83-177 MR0013421 (7:151b)

[12] Nivoche, S., Proof of a conjecture of Zahariuta concerning a problem of Kolmogorov on the ع-entropy, Invent. Math., 2004, 158, 413-450 MR2096799 (2005j:32035a)

[13] Poletsky, E., Approximation of plurisubharmonic functions by multipole Green functions, Trans. Amer. Math. Soc., 2003, 335, 1579-1591 MR1946406 (2003k:32047)

[14] Ransford, T., Potential Theory in the Complex Plane, Cambridge University Press, 1995 MR1334766 (96e:31001)

[15] Sadullaev, A., Plurisubharmonic measures and capacities on complex manifolds, Uspekhi Mat. Nauk, 1981, 36(4), 53-105 MR629683 (83c:32026)

[16] Sibony, N., Prolongement des fonctions holomorphes bornées et métrique de Carathéodory, Inventiones Mathematicae, 1975, 29, 205-230 MR0385164 (52:6029)

[17] Zahariuta, V., Spaces of analytic functions and complex potential theory, in: Linear Topological Spaces and Complex Analysis, 1994, 1, 74-146 MR1323360 (96a:46046)

[18] Zahariuta, V., On approximation by special analytic polyhedral pairs, Ann. Math. Pol., 2003, 80, 243-256 MR1972851 (2004f:32045)

FEnS, Sabanci University, 34956 Tuzla/Istanbul, Turkey

E-mail address: aytuna@sabanciuniv.edu

FEnS, Sabanci University, 34956 Tuzla/Istanbul, Turkey

E-mail address: zaha@sabanciuniv.edu 